\title{
Sharpness of the Phase Transition for the Orthant Model
}

\author{
Thomas Beekenkamp ${ }^{1}$ (D)
}

Received: 9 February 2021 / Accepted: 17 September 2021 / Published online: 15 October 2021

(C) The Author(s) 2021

\begin{abstract}
The orthant model is a directed percolation model on $\mathbb{Z}^{d}$, in which all clusters are infinite. We prove a sharp threshold result for this model: if $p$ is larger than the critical value above which the cluster of 0 is contained in a cone, then the shift from 0 that is required to contain the cluster of 0 in that cone is exponentially small. As a consequence, above this critical threshold, a shape theorem holds for the cluster of 0 , as well as ballisticity of the random walk on this cluster.
\end{abstract}

Keywords Oriented percolation · Phase transitions · Degenerate random environments · Random graphs

Mathematics Subject Classification (2010) 60K35

\section{Introduction and Main Result}

We consider the orthant model on the directed graph $\mathbb{Z}^{d}, d \geq 2$, with nearest neighbour edges. This model is informally described as follows. Let $e_{1}, \ldots, e_{d}$ be the standard unit basis vectors of $\mathbb{R}^{d}$. We set $\mathcal{E}_{+}:=\left\{e_{1}, \ldots, e_{d}\right\}$, and $\mathcal{E}_{-}:=$ $\left\{-e_{1}, \ldots,-e_{d}\right\}$, as well as $\mathcal{E}=\mathcal{E}_{+} \cup \mathcal{E}_{-}$. A vertex $v \in \mathbb{Z}^{d}$ is connected to the vertices $v+e$ for all $e \in \mathcal{E}_{+}$with a directed edge with probability $p$, independently of the other vertices. Otherwise, so with probability $1-p$, the vertex $v$ is connected to the vertices $v+e$ for all $e \in \mathcal{E}_{-}$. This model was introduced by Holmes and Salisbury $[10,11]$. The model is shown in Fig. 1 for $d=2$.

The random directed graph obtained in this way has the property that every vertex is in an infinite cluster, since, for example, either the edge in the direction $e_{1}$ or in the direction $-e_{2}$ is always available. Therefore, there is no classical percolation phase

Thomas Beekenkamp

Thomas.Beekenkamp@math.lmu.de

1 Mathematisches Institut, Ludwig-Maximilians-Universität München, Theresienstraße 39, 80333

München, Germany 


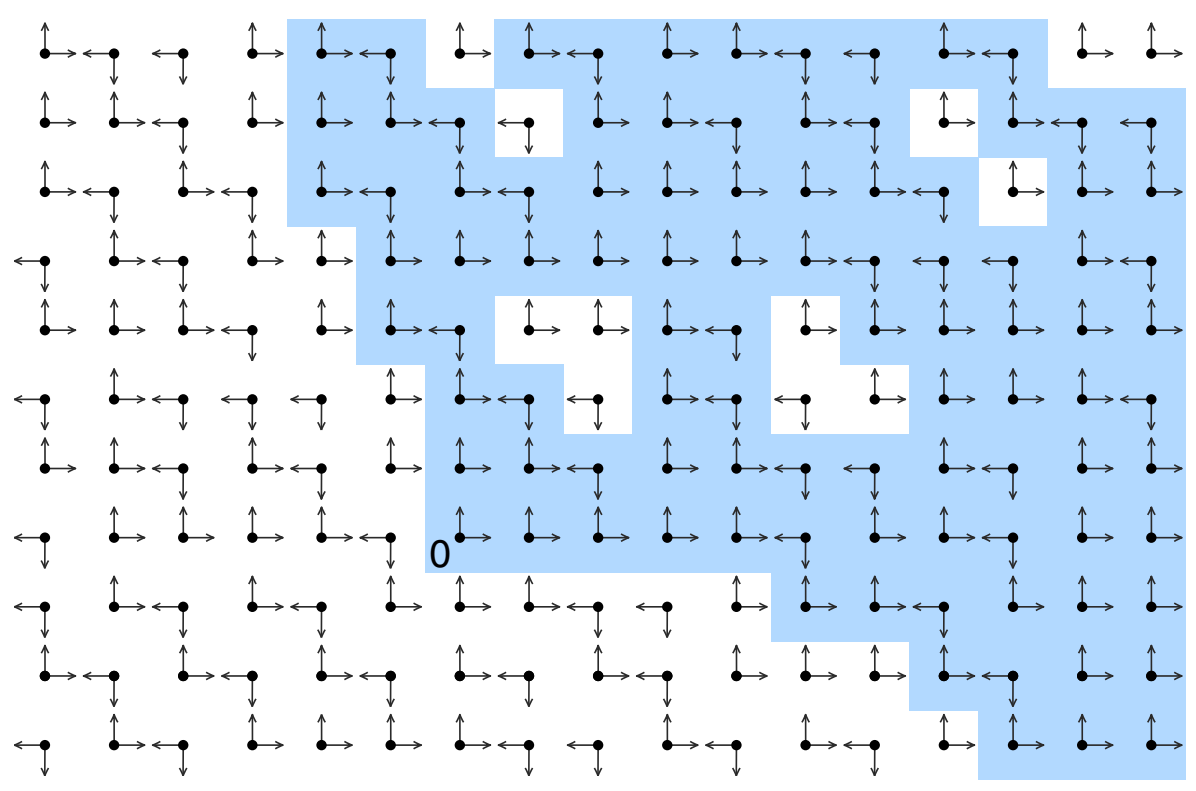

Fig. 1 The orthant model on $\mathbb{Z}^{2}$. The cluster of the origin is shaded blue

transition in this model, where for small $p$ there are only finite clusters, and for large $p$ an infinite cluster exists. Instead, for small $p$ the clusters will have a tendency to move in the directions of $\mathcal{E}_{-}$, and for large $p$ a tendency in the directions of $\mathcal{E}_{+}$. In order to make this notion precise, we introduce a cone oriented in the direction $\mathbf{1}:=e_{1}+\cdots+e_{d}$. For $0 \leq \eta \leq 1$, we define the convex cone

$$
\mathcal{K}_{\eta}=\left\{x \in \mathbb{R}^{d}: x \cdot \mathbf{1} \geq \eta\|x\|_{1}\right\} .
$$

Note that $\mathcal{K}_{0}$ is a half-space, and that $\mathcal{K}_{1}$ is the positive orthant.

For $v, w \in \mathbb{Z}^{d}$, we say that $v \longrightarrow w$, whenever there is a directed path from $v$ to $w$. Note that this is not a symmetrical event, since we are working with a directed graph. Furthermore, for $A \subset \mathbb{Z}^{d}$, we say that $v \longrightarrow A$, whenever there exists $w \in A$ such that $v \longrightarrow w$. For $v \in \mathbb{Z}^{d}$, let $\mathcal{C}_{v}$ denote the forward cluster of $v$, i.e.,

$$
\mathcal{C}_{v}:=\left\{w \in \mathbb{Z}^{d}: v \longrightarrow w\right\} .
$$

We can define the critical point above which $\mathcal{C}_{0}$ is contained in a translated cone with parameter $\eta$ :

$$
\tilde{p}_{c}(\eta):=\inf \left\{p: \mathbb{P}_{p}\left(\mathcal{C}_{0} \subset-n \mathbf{1}+\mathcal{K}_{\eta} \text { for some } n \in \mathbb{N}\right)=1\right\} .
$$

Note that this critical point is increasing in $\eta$, and that $\tilde{p}_{c}(1)=1$, so that $\tilde{p}_{c}(\eta)$ is of interest for $\eta \in[0,1)$. In fact, Holmes and Salisbury [12] have proven that $p_{c}^{\mathrm{OSP}} \leq \tilde{p}_{c}(\eta)<1$ for all $0<\eta<1$, where $p_{c}^{\mathrm{OSP}}$ is the critical parameter for oriented site percolation on the triangular lattice. Furthermore, from considerations later in this section, it follows that $\tilde{p}_{c}(0)>0$. Therefore, $\tilde{p}_{c}(\eta)$ is non-trivial for $0 \leq \eta<1$. In this paper, we will prove the following result. 
Theorem 1 Consider the orthant model on $\mathbb{Z}^{d}$ with parameter $p$. Let $0 \leq \eta<1$ and suppose $p>\tilde{p}_{c}(\eta)$. Then, there exists a constant $c_{p}>0$, such that for all $n \in \mathbb{N}$,

$$
\mathbb{P}_{p}\left(0 \longrightarrow\left(-n \mathbf{1}+\mathcal{K}_{\eta}\right)^{c}\right) \leq \exp \left(-c_{p} n\right) .
$$

The above result for $d=2$ was proven by Holmes and Salisbury [10] by making a connection with oriented site percolation on the triangular lattice. The result of Theorem 1 is known as a sharp threshold, or as a sharp phase transition. This type of result has been proven in a variety of models, most notably, and initially, for Bernoulli bond percolation in the 80's by Menshikov [16] and Aizenman and Barsky [1]. More recently, a revolutionary technique using the OSSS inequality was developed by Duminil-Copin, Raoufi and Tassion to prove sharp thresholds in models with more complexities [5-8]. These models include the random cluster model, Voronoi percolation, Boolean percolation, Gaussian fields, the corrupted compass model and the Widom-Rowlinson model [3, 4, 15, 17].

As a consequence of the above sharp threshold result, we can prove a shape theorem for $\mathcal{C}_{0}$ above $\tilde{p}_{c}:=\lim _{\eta \downarrow 0} \tilde{p}_{c}(\eta)$. This critical point can also be written as

$$
\tilde{p}_{c}=\inf \left\{p: \exists \eta>0 \text { s.t. } \mathbb{P}_{p}\left(\mathcal{C}_{0} \subset-n \mathbf{1}+\mathcal{K}_{\eta} \text { for some } n \in \mathbb{N}\right)=1\right\} \text {. }
$$

A shape theorem for the orthant model was first proven by Holmes and Salisbury [14] for large $p$. Using Theorem 1, we can extend this result to all $p>\tilde{p}_{c}$. In order to state the shape theorem, we introduce for $u \in \mathbb{Z}^{d}$

$$
\beta_{n}(u):=\inf \left\{k \in \mathbb{Z}: k \mathbf{1}+n u \in \mathcal{C}_{0}\right\} .
$$

Furthermore, let $\Lambda_{r}:=\left\{v \in \mathbb{Z}^{d}:\|v\|_{\infty} \leq r\right\}$ be the closed ball around 0 with radius $r$ with respect to the $L^{\infty}$-norm. Borrowing the notation from [14], the shape theorem for the orthant model can be stated as follows.

Corollary 1 (Shape theorem) Let $p>\tilde{p}_{c}$. The following hold for the orthant model on $\mathbb{Z}^{d}$ with parameter $p$.

a) For $u \in \mathbb{Z}^{d}$, there is a deterministic $\gamma(u) \in \mathbb{R}$ such that $\frac{\beta_{n}(u)}{n} \rightarrow \gamma(u)$, as $n \rightarrow \infty, \mathbb{P}_{p}$-almost surely.

b) This limit satisfies $\gamma(u+w) \leq \gamma(u)+\gamma(w), \gamma(r u)=r \gamma(u), \gamma(u+r \mathbf{1})=\gamma(u)-$ $r$, for $u, w \in \mathbb{Z}^{d}$, and $r \in \mathbb{N}$. Furthermore, $\gamma$ is symmetric under permutation of coordinates, $\gamma(u) \geq 0$ if $u \cdot \mathbf{1} \leq 0$, and $\gamma(u) \leq 0$ if $u$ lies in the positive orthant.

c) The limit $\gamma$ extends to a Lipschitz map $\mathbb{R}^{d} \rightarrow \mathbb{R}$ with these same properties, but for $r \in[0, \infty)$ and $u, w \in \mathbb{R}^{d}$.

d) The set $C:=\left\{z \in \mathbb{R}^{d}: \gamma(z) \leq 0\right\}$ is a closed convex cone, which is symmetric under permutations of the coordinates, contains the positive orthant, and is contained in the half-space $\mathcal{K}_{0}=\{z: z \cdot \mathbf{1} \geq 0\}$.

e) Let $\mathcal{C}_{0}^{*}:=\mathcal{C}_{0}+e_{1} \mathbb{N}_{0}$, i.e., " $\mathcal{C}_{0}$ with its holes filled in". It holds that $\frac{1}{n} \mathcal{C}_{0}^{*} \rightarrow C$, in the sense that for every $\varepsilon>0$ and every $r>0$, the following holds $\mathbb{P}_{p}$-a.s. for sufficiently large (random) $n$ :

$$
\left(\Lambda_{r} \cap \frac{1}{n} \mathcal{C}_{0}^{*}\right) \subset \Lambda_{\varepsilon}+C, \quad \text { and }\left(\Lambda_{r} \cap C\right) \subset \Lambda_{\varepsilon}+\frac{1}{n} \mathcal{C}_{0}^{*}
$$


To prove this theorem for all $p>\tilde{p}_{c}$, we modify the proof in [14] by using Theorem 1 in the places where they require $p$ to be large. Another consequence of Theorem 1 is the ballisticity of the random walk on $\mathcal{C}_{0}$.

Corollary 2 (Ballisticity of the Random Walk) Consider the orthant model on $\mathbb{Z}^{d}$ with parameter $p>\tilde{p}_{c}$. Let $X_{n}$ be a simple random walk on $\mathcal{C}_{0}$ and let $P$ be the annealed law of this random walk (i.e., averaged over $\mathcal{C}_{0}$ ). Then there exists $v>0$ such that $\frac{1}{n} X_{n} \rightarrow v \mathbf{1} P$-a.s. as $n \rightarrow \infty$, and

$$
\left(\frac{X_{\lfloor n t\rfloor}-v \mathbf{1} n t}{\sqrt{n}}\right)_{t \geq 0} \Rightarrow\left(B_{t}\right)_{t \geq 0}, \quad \text { as } n \rightarrow \infty,
$$

weakly under $P$, in the space of càdlàg functions endowed with the Skorohod topology, where $\left(B_{t}\right)_{t \geq 0}$ is a d-dimensional Brownian motion with nonsingular covariance matrix $\Sigma$.

This is Theorem 1.4 combined with Corollary 1.9 of [12] by Holmes and Salisbury applied to the orthant model. Their theorem is stated for more general models, and requires two conditions, one of which they show to hold for the orthant model with any value of $p$. The other condition is the existence of $\eta>0$ and $c>0$ such that $\mathbb{P}_{p}\left(0 \longrightarrow\left(-n \mathbf{1}+\mathcal{K}_{\eta}\right)^{c}\right) \leq \exp \left(-c n^{\beta}\right)$, for some $\beta>0$. By taking $\beta=1$ and assuming $p>\tilde{p}_{c}$, it follows from Theorem 1 that this condition holds for the orthant model with parameter $p$. Corollary 2 is therefore an immediate consequence of combining Theorem 1 with Theorem 1.4 of [12].

Despite the above results, the theoretical picture of the orthant model is still incomplete. We will use the remainder of this section to formulate two open questions for the model. The shape theorem and the ballisticity of the random walk have now been shown to hold for $p>\tilde{p}_{c}:=\lim _{\eta \downarrow 0} \tilde{p}_{c}(\eta)$. A natural extension would be to prove these results for $p>\tilde{p}_{c}(0)$. This would follow from the continuity of $\tilde{p}_{c}(\eta)$.

Open Problem 1 Consider the orthant model on $\mathbb{Z}^{d}$. The function $\eta \mapsto \tilde{p}_{c}(\eta)$ is continuous on $[0,1]$.

A critical value other than $\tilde{p}_{c}$ can be defined for the orthant model. In order to state this definition, we introduce for $v \in \mathbb{Z}^{d}$,

$$
L_{v}:=\inf \left\{k \in \mathbb{Z}: v+k e_{1} \in \mathcal{C}_{0}\right\}
$$

The critical value $p_{c}$ is defined as

$$
p_{c}:=\sup \left\{p: L_{0}=-\infty \text { a.s. }\right\}
$$

Holmes and Salisbury [13] have shown that this critical value is nontrivial, i.e., $0<$ $p_{c}<1$. From the definitions of the critical values, it is clear that $p_{c} \leq \tilde{p}_{c}(0) \leq \tilde{p}_{c}$. However, it is as of yet unclear that above $p_{c}$ there exists a cone with parameter $\eta>0$ that contains the forward cluster of 0 . 
Open Problem 2 Consider the orthant model on $\mathbb{Z}^{d}$. It holds that

$$
p_{c}=\tilde{p}_{c}
$$

In order to prove this, perhaps it is most natural to first show that $p_{c}=\tilde{p}_{c}(0)$, and subsequently show the continuity of $\tilde{p}_{c}(\eta)$.

The sharp threshold result of Theorem 1 will be proven in Section 3, while some preliminaries required for this proof are introduced in Section 2. The proof for Corollary 1 is given in Section 4.

\section{Preliminaries}

We can couple the model for different values of $p$ by considering a coupling similar to the standard coupling in Bernoulli percolation: we consider a family of i.i.d. random variables $\left(U_{v}\right)_{v \in \mathbb{Z}^{d}}$, and connect $v$ to $v+e$ for all $e \in \mathcal{E}_{+}$whenever $U_{v}<p$, and to $v+e$ for all $e \in \mathcal{E}_{-}$if $U_{v} \geq p$. One difficulty in analysing the orthant model is the lack of monotonicity in $p$, i.e., a path from $v$ to $w$ might be lost if we increase $p$. To deal with this issue, we introduce the half-orthant model. In this model a vertex $v$ is always connected to $v+e$ for all $e \in \mathcal{E}_{+}$, whereas $v$ is connected to $v+e$, for all $e \in \mathcal{E}_{-}$, with probability $1-p$. This model is monotone in $p$, in the sense that $\mathbb{1}\{v \longrightarrow w\}$ is monotonically decreasing in $p$, under the coupling where $v$ is connected to $v+e$, for all $e \in \mathcal{E}_{-}$, whenever $U_{v}>p$. Let $\mathcal{C}_{v}^{*}$ denote the forward cluster of $v$ in the half-orthant model. The half-orthant model dominates the orthant model, in the sense that $\mathcal{C}_{v} \subseteq \mathcal{C}_{v}^{*}$, almost surely under a suitable coupling between the two models. For $v \in \mathbb{Z}^{d}$, we further define

$$
L_{v}^{*}:=\inf \left\{k \in \mathbb{Z}: v+k e_{1} \in \mathcal{C}_{0}^{*}\right\}
$$

From the domination it follows that $L_{v} \geq L_{v}^{*}$. However, it turns out that equality holds: $L_{v}^{*}=L_{v}$ for all $v \in \mathbb{Z}^{d}[13$, Thm. 1.9]. So, loosely speaking, if we only care about the leftmost boundary of $\mathcal{C}_{0}$, it does not matter if we consider the orthant model or the half-orthant model. This allows us to prove statements for the orthant model by making use of the monotonicity of the half orthant model. In light of this, we remark that the above definition of $\mathcal{C}_{0}^{*}$ coincides with the definition stated in Corollary 1. Furthermore, we note that $L_{v}<\infty$ for all $v \in \mathbb{Z}^{d}$, since $L_{v}^{*}<\infty$, but it might be the case that $L_{v}=-\infty$ for some $v \in \mathbb{Z}^{d}$. In fact, Holmes and Salisbury proved that if $L_{v}$ is finite for some $v \in \mathbb{Z}^{d}$, then it is finite for all $v \in \mathbb{Z}^{d}$ (Lemma 2.2 of [13]). For $p<p_{c}$ it follows that $L_{v}^{*}=-\infty$ for all $v \in \mathbb{Z}^{d}$, and in this case, $\mathcal{C}_{0}^{*}=\mathbb{Z}^{d}$. On the other hand, if $p>p_{c}, L_{v}$ is finite for all $v \in \mathbb{Z}^{d}$ using the monotonicity of the half-orthant model.

To prove Theorem 1, it therefore suffices to work with the half-orthant model. We start by giving a formal description of this model. For $p \in[0,1]$, we consider the probability space $\left(\Omega, \mathcal{F}, \mathbb{P}_{p}\right)$, where

$$
\Omega=\{0,1\}^{\mathbb{Z}^{d}}
$$


the $\sigma$-algebra $\mathcal{F}$ is generated by the cylindrical events, and $\mathbb{P}_{p}$ is the product measure on $\Omega$ such that $\mathbb{P}_{p}\left(\omega_{v}=1\right)=p$ for all $v \in \mathbb{Z}^{d}$. From $\omega \in \Omega$ we obtain the edge configuration $\xi \subseteq\left\{(v, v+e): v \in \mathbb{Z}^{d}, e \in \mathcal{E}\right\}$ by adding the edge $(v, v+e)$ to the graph for all $e \in \mathcal{E}_{+}$, and for all $e \in \mathcal{E}_{-}$whenever $\omega_{v}=0$.

For $v, w \in \mathbb{Z}^{d}$, we say $v \sim w$ when $v$ is a neighbour of $w$, i.e., whenever $w=v+e$ for some $e \in \mathcal{E}$. Furthermore, we say that $v \sim w$, whenever $(v, w) \in \xi$. For $A \subset \mathbb{Z}^{d}$, we say that $v \stackrel{A}{\longrightarrow} w$, whenever there is a path from $v$ to $w$ using only edges in $\xi$ with starting points in $A$. Note that $w$ does not have to be an element $A$ for this event to hold. For $A=\mathbb{Z}^{d}$ we use the shorthand notation $\{v \longrightarrow w\}:=\left\{v \stackrel{\mathbb{Z}^{d}}{\longrightarrow} w\right\}$. Furthermore, the event $v \stackrel{A}{\longrightarrow} v$ trivially holds for all $v \in \mathbb{Z}^{d}$, and all $A \subset \mathbb{Z}^{d}$.

The proof will make use of the OSSS inequality for Boolean functions $f: \Omega \rightarrow$ $\{0,1\}$. In order to state this inequality, we introduce the influence of $v$ on $f$. This is defined as

$$
\operatorname{Inf}_{v}:=\mathbb{P}_{p}\left(f(\omega) \neq f\left(\omega^{v \mapsto p}\right)\right),
$$

where $\omega^{v \mapsto p}$ is given by

$$
\left(\omega^{v \mapsto p}\right)_{w}= \begin{cases}\omega_{v}^{\prime} & \text { if } w=v, \\ \omega_{v} & \text { if } w \neq v,\end{cases}
$$

for a Bernoulli distributed random variable $\omega_{v}^{\prime}$ with parameter $p$, which is independent of the other variables. In other words,

$$
\operatorname{Inf}_{v}=2 p(1-p) \mathbb{P}_{p}(v \text { is pivotal for the event }\{f=1\}) .
$$

A decision tree $T$ is a random sequence of vertices $\left(v_{0}, v_{1}, \ldots\right)$ that is built sequentially as follows. The tree starts by revealing the value of $\omega_{v_{0}}$, for the starting vertex $v_{0}$. Then, depending on the value of $\omega_{v_{0}}$, it chooses a vertex $v_{1}$ and reveals the value of $\omega_{v_{1}}$. This process continues until it has obtained enough information to determine the value of $f$, i.e., whenever the values of $\omega_{v}$ for unrevealed vertices $v$, cannot change $f$ any more. This leads to the definition of the revealment of $v$ by $T$ :

$$
\operatorname{Rev}_{v}(T):=\mathbb{P}_{p}\left(\omega_{v} \text { is revealed by } T\right) .
$$

The OSSS inequality states that for a Boolean function $f$ depending on finitely many variables and a decision tree $T$ that determines the value of $f$ we have

$$
\operatorname{Var}_{p}(f) \leq \sum_{v \in \mathbb{Z}^{d}} \operatorname{Inf}_{v} \operatorname{Rev}_{v}(T) .
$$

This inequality was proven by O'Donnell, Saks, Schramm and Servedio [19]. A detailed exposition of Boolean functions has been written by O'Donnell [18], in which the proof of the OSSS inequality can also be found. Still, we will make use of Boolean functions $f$ that depend on infinitely many variables, so that we require an additional limit argument. The OSSS inequality can be generalized to Boolean functions on $\Omega$, provided $f$ is measurable with respect to the $\sigma$-algebra

$$
\sigma\left(\left\{\omega_{v}: \omega_{v} \text { revealed by } T\right\}\right)
$$


This is for example the case for decision trees that terminate in a finite number of steps on the set $\{f=1\}$. This generalisation of the OSSS inequality has been stated by Duminil-Copin, Raoufi and Tassion [7]. A proof can be found in [2].

\section{Proof of Theorem 1}

For $\eta \geq 0$ and $n \in \mathbb{N}$, we define the Boolean function

$$
f_{n}:=\mathbb{1}\left\{0 \longrightarrow\left(-n \mathbf{1}+\mathcal{K}_{\eta}\right)^{c}\right\} .
$$

\subsection{Exploration Algorithm}

We now introduce decision trees that determine the value of $f_{n}$. A vital point in the proof is that we can uniformly bound the revealment of the vertices. If we only use one decision tree with a deterministic starting point, then the starting vertex will have revealment 1 , so that we cannot find a nontrivial uniform bound on the revealment. Therefore, we will introduce the decision trees $T_{k}$, for $1 \leq k \leq n$, which all start at different vertices. In this way, we can average over $k$ and have a meaningful uniform bound on the revealment. The basic idea of the decision tree $T_{k}$ is that it explores the cluster of the boundary of $-k \mathbf{1}+\mathcal{K}_{\eta}$. If $0 \longrightarrow\left(-n \mathbf{1}+\mathcal{K}_{\eta}\right)^{c}$, this path must go through the boundary of the cone $-k \mathbf{1}+\mathcal{K}_{\eta}$, so that $T_{k}$ determines $f_{n}$. Furthermore, $T_{k}$ terminates in a finite number of steps when $f_{n}=1$.

We will now describe the exploration algorithm of $T_{k}$ more precisely. We define the boundary and the outer boundary of the cone as

$$
\begin{aligned}
\partial\left(-k \mathbf{1}+\mathcal{K}_{\eta}\right) & :=\left\{v \in\left(-k \mathbf{1}+\mathcal{K}_{\eta}\right) \cap \mathbb{Z}^{d}: \exists w \in\left(-k \mathbf{1}+\mathcal{K}_{\eta}\right)^{c} \cap \mathbb{Z}^{d} \text { with } v \sim w\right\}, \\
\partial^{+}\left(-k \mathbf{1}+\mathcal{K}_{\eta}\right) & :=\left\{v \in\left(-k \mathbf{1}+\mathcal{K}_{\eta}\right)^{c} \cap \mathbb{Z}^{d}: \exists w \in\left(-k \mathbf{1}+\mathcal{K}_{\eta}\right) \cap \mathbb{Z}^{d} \text { with } v \sim w\right\} .
\end{aligned}
$$

The decision tree $T_{k}$ consists of two phases. In the first phase, $T_{k}$ explores the backward cluster of $\partial\left(-k \mathbf{1}+\mathcal{K}_{\eta}\right)$ inside the cone, that is, it explores the set $\{v \in$ $\left.-k \mathbf{1}+\mathcal{K}_{\eta}: v \longrightarrow \partial\left(-k \mathbf{1}+\mathcal{K}_{\eta}\right)\right\}$. When this is finished, the set of vertices

$$
\left\{v \in \partial^{+}\left(-k \mathbf{1}+\mathcal{K}_{\eta}\right): 0 \stackrel{-k \mathbf{1}+\mathcal{K}_{\eta}}{\longrightarrow} v\right\}
$$

has been determined. In the second phase, the algorithm explores the forward clusters of these vertices. If for one of these vertices we find that $v \longrightarrow\left(-n \mathbf{1}+\mathcal{K}_{\eta}\right)^{c}$, then we also have $0 \longrightarrow\left(-n \mathbf{1}+\mathcal{K}_{\eta}\right)^{c}$. A schematic visualisation of the algorithm is shown in Fig. 2.

There is however one technical issue: since $f_{n}$ depends on the state of infinitely many vertices, it is possible that the algorithm gets stuck exploring inside $-k \mathbf{1}+\mathcal{K}_{\eta}$, and never gets to explore the forward clusters outside $-k \mathbf{1}+\mathcal{K}_{\eta}$. In order to deal with this, the decision tree operates in rounds, denoted by $i \in \mathbb{N}$. Recall that $\Lambda_{r}$ is the ball of radius $r$ around 0 with respect to $L^{\infty}$-norm. In round $i$ we only explore inside $\Lambda_{i}$, so it is not possible to get stuck in any particular phase. Note that if $0 \longrightarrow$ $\left(-n \mathbf{1}+\mathcal{K}_{\eta}\right)^{c}$, there exists $i \in \mathbb{N}$ such that $0 \stackrel{\Lambda_{i}}{\longrightarrow}\left(-n \mathbf{1}+\mathcal{K}_{\eta}\right)^{c}$. 
THOMAS BEEKENKAMP

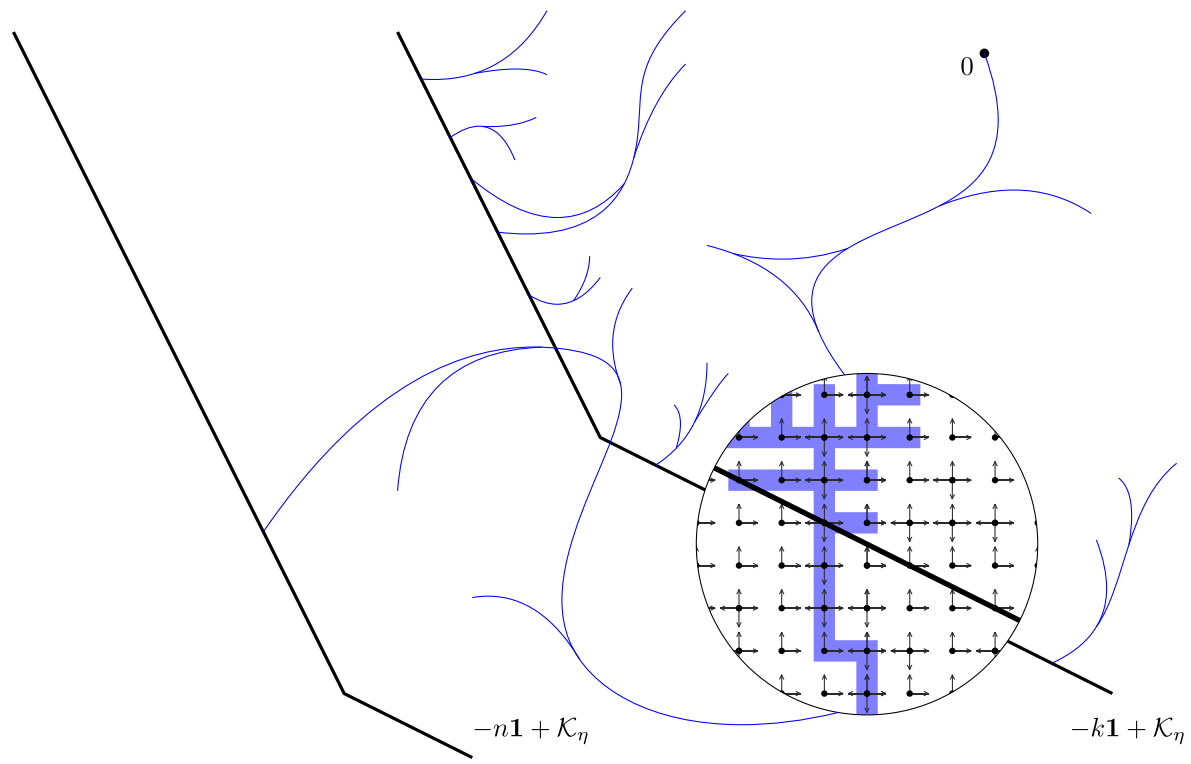

Fig. 2 The algorithm $T_{k}$ exploring the cluster of $\partial\left(-k \mathbf{1}+\mathcal{K}_{\eta}\right)$ to find a path from 0 to $\left(-n \mathbf{1}+\mathcal{K}_{\eta}\right)^{c}$. The blue vertices are revealed.

We denote by $\mathcal{R}$ the set of revealed vertices. Furthermore, we denote by $\mathcal{A}$ the set of active vertices for the first phase and by $\mathcal{B}$ the set of active vertices for the second phase. We start the algorithm by setting $\mathcal{A}:=\mathcal{A}_{0}:=\partial^{+}\left(-k \mathbf{1}+\mathcal{K}_{\eta}\right)$, and $\mathcal{B}:=\varnothing$. The pseudocode of $T_{k}$ is given in Algorithm 1. We have to be careful when updating $\mathcal{A}$ in the first phase: by revealing $v$ it is possible that we create a new path $x \stackrel{\mathcal{R}}{\longrightarrow} \partial^{+}\left(-k \mathbf{1}+\mathcal{K}_{\eta}\right)$ for some $x \neq v$. Therefore, it is not sufficient to only consider $w \sim v$ for the update of $\mathcal{A}$. Instead, we add $w$ to $\mathcal{A}$ if and only if $w \notin \mathcal{R} \cap \mathcal{B}$, and if there exists $x \in \mathcal{R}$ such that $x \sim w$, and $x \stackrel{\mathcal{R}}{\longrightarrow} \partial^{+}\left(-k \mathbf{1}+\mathcal{K}_{\eta}\right)$. At the start of any iteration of the inner loops of the algorithm, the following hold for the active sets $\mathcal{A}$ and $\mathcal{B}$ :

$$
\begin{aligned}
& \mathcal{A} \subseteq \mathcal{A}_{0} \backslash \mathcal{R} \cup\left\{v \in\left(-k \mathbf{1}+\mathcal{K}_{\eta}\right) \cap \mathbb{Z}^{d}: v \notin \mathcal{R}, \exists w \sim v, \text { s.t. } w \stackrel{\mathcal{R}}{\longrightarrow} \partial^{+}\left(-k \mathbf{1}+\mathcal{K}_{\eta}\right)\right\}, \\
& \mathcal{B}=\left\{v \in\left(-n \mathbf{1}+\mathcal{K}_{\eta}\right) \cap \mathbb{Z}^{d}: v \notin \mathcal{R}, \exists w \in \partial^{+}\left(-k \mathbf{1}+\mathcal{K}_{\eta}\right) \text { s.t. } 0 \stackrel{\mathcal{R}}{\longrightarrow} w, w \stackrel{\mathcal{R}}{\longrightarrow} v\right\} .
\end{aligned}
$$

\subsection{Bound on the Revealment}

Let $\theta_{n}(p):=\mathbb{P}_{p}\left(f_{n}=1\right)$. Summing the OSSS inequality over $k$ gives

$$
n \theta_{n}(p)\left(1-\theta_{n}(p)\right) \leq \sum_{v \in \mathbb{Z}^{d}} \operatorname{Inf}_{v} \sum_{k=1}^{n} \operatorname{Rev}_{v}\left(T_{k}\right)
$$

We will now bound $\sum_{k=1}^{n} \operatorname{Rev}_{v}\left(T_{k}\right)$ uniformly in $v$. Let $k_{v}$ be such that $v \in$ $\partial^{+}\left(-k_{v} \mathbf{1}+\mathcal{K}_{\eta}\right)$. Note that $k_{v-1}=k_{v}+1$. Suppose first that $k>k_{v}+1$. If $v$ is 
revealed by $T_{k}$ in the second phase, we have $0 \longrightarrow \partial^{+}\left(-k \mathbf{1}+\mathcal{K}_{\eta}\right)$ by (1). On the other hand, if $v$ is revealed by $T_{k}$ in the first phase, there exists $w \sim v$ such that $w \longrightarrow \partial^{+}\left(-k \mathbf{1}+\mathcal{K}_{\eta}\right)$. Since $k>k_{v}+1$, we know that $v-\mathbf{1} \in-k \mathbf{1}+\mathcal{K}_{\eta}$, which implies that every neighbour of $v$ is also contained in $-k \mathbf{1}+\mathcal{K}_{\eta}$. Applying the union bound gives

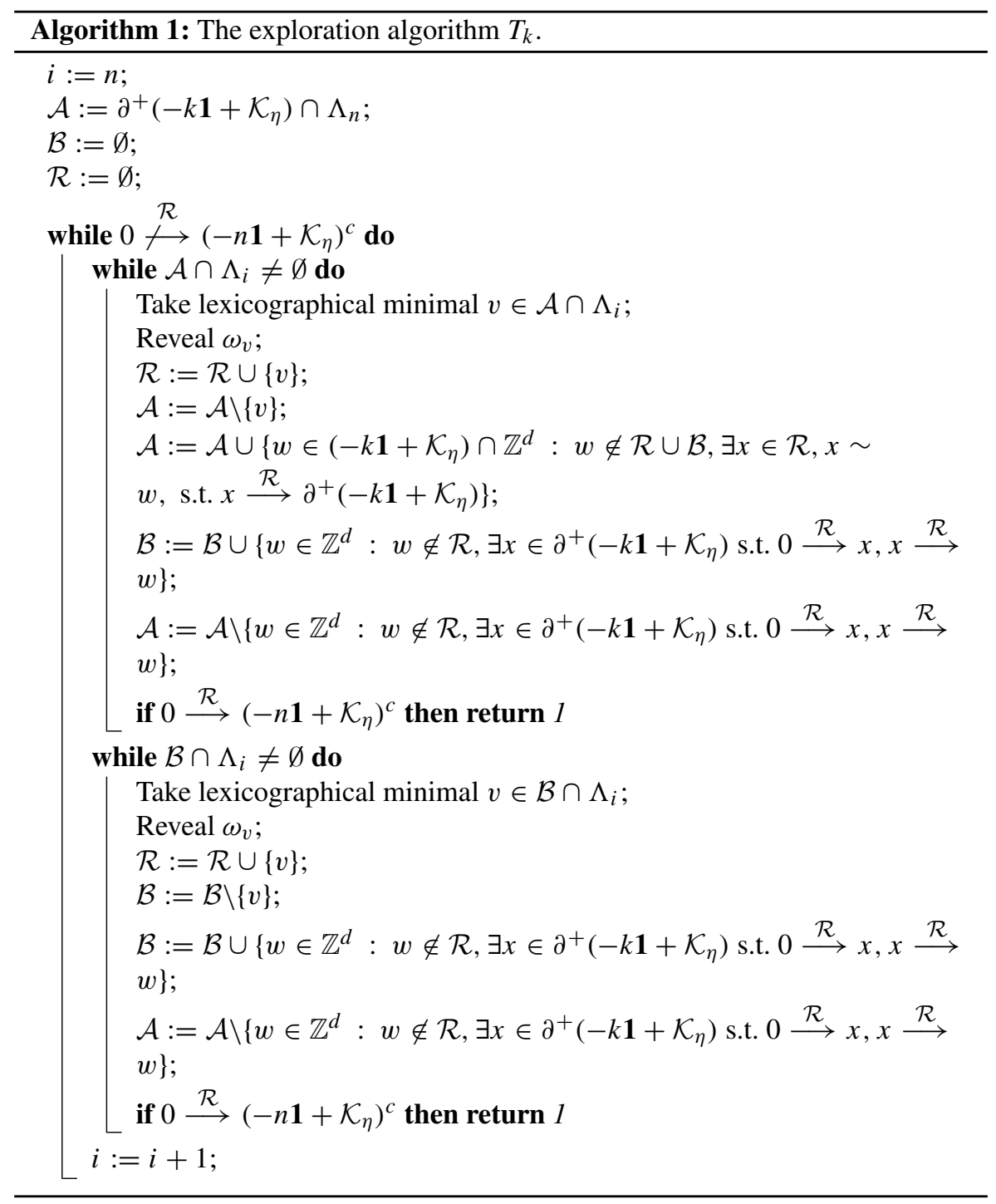

$$
\sum_{k=1}^{n} \mathbb{1}\left\{k>k_{v}+1\right\} \operatorname{Rev}_{v}\left(T_{k}\right) \leq \sum_{k=1}^{n} \mathbb{1}\left\{k>k_{v}+1\right\}\left(\theta_{k}(p)+\sum_{w \sim v} \mathbb{P}_{p}\left(w \longrightarrow \partial^{+}\left(-k \mathbf{1}+\mathcal{K}_{\eta}\right)\right)\right) .
$$

Let

$$
d_{k}^{w}:=\sup \left\{l \in \mathbb{Z}: w-l \mathbf{1} \in-k \mathbf{1}+\mathcal{K}_{\eta}\right\} .
$$


Note that $d_{k}^{w} \geq 0$ for all $k>k_{v}+1$. Furthermore $w-d_{k}^{w} \mathbf{1} \in-k \mathbf{1}+\mathcal{K}_{\eta}$, and

$$
w-d_{k}^{w} \mathbf{1}+\mathcal{K}_{\eta} \subseteq-k \mathbf{1}+\mathcal{K}_{\eta}
$$

since $-k \mathbf{1}+\mathcal{K}_{\eta}$ is a convex cone. Therefore, using translation invariance, it follows that

$$
\begin{aligned}
\sum_{k=1}^{n} \mathbb{1}\left\{k>k_{v}+1\right\} \operatorname{Rev}_{v}\left(T_{k}\right) & \leq \sum_{k=1}^{n} \mathbb{1}\left\{k>k_{v}+1\right\}\left(\theta_{k}(p)+\sum_{w \sim v} \mathbb{P}_{p}\left(w \longrightarrow \partial^{+}\left(w-d_{k}^{w} \mathbf{1}+\mathcal{K}_{\eta}\right)\right)\right) \\
& =\sum_{k=1}^{n} \mathbb{1}\left\{k>k_{v}+1\right\}\left(\theta_{k}(p)+\sum_{w \sim v} \mathbb{P}_{p}\left(0 \longrightarrow \partial^{+}\left(-d_{k}^{w} \mathbf{1}+\mathcal{K}_{\eta}\right)\right)\right) .
\end{aligned}
$$

We have $d_{k_{v}+2}^{w} \geq 0$, and using the fact that $d_{k+1}^{w}=d_{k}^{w}+1$, we know $d_{k_{v}+l}^{w} \geq l-2$, for all $l \geq 2$. We can thus bound

$$
\begin{aligned}
\sum_{k=1}^{n} \mathbb{1}\left\{k>k_{v}+1\right\} \operatorname{Rev}_{v}\left(T_{k}\right) & \leq \sum_{k=1}^{n} \theta_{k}(p)+2 d \sum_{k=0}^{n-1} \mathbb{P}_{p}\left(0 \longrightarrow \partial^{+}\left(-k \mathbf{1}+\mathcal{K}_{\eta}\right)\right) \\
& =\sum_{k=1}^{n} \theta_{k}(p)+2 d \sum_{k=0}^{n-1} \theta_{k}(p)
\end{aligned}
$$

Now suppose $k<k_{v}$, so $v \notin-k \mathbf{1}+\mathcal{K}_{\eta} \cup \partial^{+}\left(-k \mathbf{1}+\mathcal{K}_{\eta}\right)$. If $v$ is revealed, it holds that $0 \stackrel{\mathcal{R}}{\longrightarrow} v$. In particular, we have $0 \longrightarrow \partial^{+}\left(-k \mathbf{1}+\mathcal{K}_{\eta}\right)$. We find

$$
\sum_{k=1}^{n} \mathbb{1}\left\{k<k_{v}\right\} \operatorname{Rev}_{v}\left(T_{k}\right) \leq \sum_{k=1}^{n} \theta_{k}(p) .
$$

Combining (4) and (5) gives

$$
\sum_{k=1}^{n} \operatorname{Rev}_{v}\left(T_{k}\right) \leq 2+2 \sum_{k=1}^{n} \theta_{k}(p)+2 d \sum_{k=0}^{n} \theta_{k}(p)=(2 d+2) \sum_{k=0}^{n} \theta_{k}(p)
$$

Writing $S_{n}:=\sum_{k=0}^{n} \theta_{k}(p)$, gives

$$
\sum_{v \in \mathbb{Z}^{d}} \operatorname{Inf}_{v} \geq \frac{1}{2 d+2} \frac{n}{S_{n}} \theta_{n}(p)\left(1-\theta_{n}(p)\right)
$$

\subsection{Analysis of the Differential Inequality}

We are now able to complete the proof of Theorem 1 . We can obtain a differential inequality by using Russo's formula. However, since $f_{n}$ depends on infinitely many vertices, $\theta_{n}(p)$ is not necessarily differentiable in $p$. Instead we have to work with the upper-right Dini derivative:

$$
D^{+} \theta_{n}(p):=\limsup _{h \downarrow 0} \frac{\theta_{n}(p+h)-\theta_{n}(p)}{h} .
$$


Using the fact that $0 \longrightarrow\left(-n \mathbf{1}+\mathcal{K}_{\eta}\right)^{c}$ is a decreasing event, i.e., $f_{n}$ is a decreasing function of $\omega$, Russo's formula gives

$$
-D^{+} \theta_{n}(p) \geq \sum_{v \in \mathbb{Z}^{d}} \mathbb{P}_{p}\left(v \text { is pivotal for }\left\{f_{n}=1\right\}\right)=\frac{1}{2 p(1-p)} \sum_{v \in \mathbb{Z}^{d}} \operatorname{Inf}_{v} .
$$

This version of Russo's formula can be found in the book on Percolation by Grimmett [9]. This is the point in the proof where we use the monotonicity of the half-orthant model, as well as the coupling given at the start of Section 2. Combining the above inequality with (6) gives

$$
-D^{+} \theta_{n}(p) \geq \frac{1}{2 d} \frac{n}{S_{n}} \theta_{n}(p)\left(1-\theta_{n}(p)\right),
$$

where we use $(2 d+2) 2 p(1-p) \leq 2 d$ for simplicity. The rest of the proof consists of analysing the above differential inequality. This analysis follows the line of DuminilCopin, Raoufi and Tassion [7], but since it differs on several points, we choose to include it. We have to work with Dini derivatives instead of regular derivatives, and, more importantly, in our case we cannot give a simple lower bound on $1-\theta_{n}(p)$.

To analyse the differential inequality, we introduce the auxiliary critical point

$$
\hat{p}_{c}(\eta):=\sup \left\{p: \limsup _{n \rightarrow \infty} \frac{\log S_{n}(p)}{\log n}=1\right\} .
$$

Note that by the monotonicity of the model, $\lim \sup _{n \rightarrow \infty} \frac{\log S_{n}(p)}{\log n}=1$ for all $p<$ $\hat{p}_{c}(\eta)$, and $\lim \sup _{n \rightarrow \infty} \frac{\log S_{n}(p)}{\log n}<1$ for all $p>\hat{p}_{c}(\eta)$. We will first show that $\hat{p}_{c}(\eta) \leq \tilde{p}_{c}(\eta)$, for $\eta \geq 0$. To prove this, we assume the contrary, and let $p \in$ $\left(\tilde{p}_{c}(\eta), \hat{p}_{c}(\eta)\right)$. Since $p>\tilde{p}_{c}(\eta)$, we can fix $l \in \mathbb{N}$, such that for all $n>l$ it holds that $1-\theta_{n}(p) \geq 1 / 2$. We define $T_{n}(p):=\frac{1}{\log n} \sum_{k=l}^{n} \frac{\theta_{k}(p)}{k}$. Taking the upper-right Dini derivative and using (7) gives

$$
-D^{+} T_{n} \geq \frac{1}{2 d} \frac{1}{\log n} \sum_{k=l}^{n} \frac{\theta_{k}(p)}{S_{k}}\left(1-\theta_{k}(p)\right) \geq \frac{1}{4 d} \frac{1}{\log n} \sum_{k=l}^{n} \frac{\theta_{k}(p)}{S_{k}} \geq \frac{1}{4 d} \frac{\log S_{n+1}-\log S_{l}}{\log n},
$$

where in the last inequality we used

$$
\frac{\theta_{k}(p)}{S_{k}} \geq \int_{S_{k}}^{S_{k+1}} \frac{1}{x} \mathrm{~d} x=\log S_{k+1}-\log S_{k} .
$$

Now let $p_{1} \in\left(p, \hat{p}_{c}(\eta)\right)$. We will integrate the differential inequality between $p$ and $p_{1}$ and use the following result regarding Dini derivatives: the Dini derivative of a decreasing function $f:[a, b] \rightarrow \mathbb{R}$ satisfies

$$
f(b)-f(a) \leq \int_{a}^{b} D^{+} f(x) \mathrm{d} x .
$$

Applying this to $T_{n}(p)$ and using (8) gives

$$
T_{n}\left(p_{1}\right)-T_{n}(p) \leq \int_{p}^{p_{1}} D^{+} T_{n}(s) \mathrm{d} s \leq-\left(p_{1}-p\right) \frac{1}{4 d} \frac{\log S_{n+1}\left(p_{1}\right)-\log S_{l}(p)}{\log n} .
$$


Furthermore, $T_{n}(p)$ converges to $\theta(p):=\lim _{n \rightarrow \infty} \theta_{n}(p)$ for $n \rightarrow \infty$, since for all $l<m<n$ :

$$
\theta_{n}(p) \frac{\sum_{k=l}^{n} \frac{1}{k}}{\log n} \leq T_{n}(p) \leq \theta_{m}(p) \frac{\sum_{k=m}^{n} \frac{1}{k}}{\log n}+\frac{\sum_{k=l}^{m-1} \frac{1}{k}}{\log n},
$$

from which the limit follows by first taking $n \rightarrow \infty$, and then $m \rightarrow \infty$. We find

$$
\theta\left(p_{1}\right)-\theta(p) \leq-\left(p_{1}-p\right) \frac{1}{4 d} \limsup _{n \rightarrow \infty} \frac{\log S_{n+1}\left(p_{1}\right)-\log S_{l}(p)}{\log n}
$$

Since $p<\hat{p}_{c}(\eta)$, we have that $\limsup _{n \rightarrow \infty} \frac{\log S_{n}(p)}{\log n}=1$ and the same holds for $p_{1}$, so that also

$$
\limsup _{n \rightarrow \infty} \frac{\log S_{n+1}(p)-\log S_{l}\left(p_{1}\right)}{\log n}=1 \text {. }
$$

We conclude

$$
\theta(p) \geq \theta(p)-\theta\left(p_{1}\right) \geq \frac{p_{1}-p}{4 d}>0,
$$

which contradicts $p>\tilde{p}_{c}(\eta)$, so that we have established that $\hat{p}_{c}(\eta) \leq \tilde{p}_{c}(\eta)$.

Now suppose $p>\hat{p}_{c}(\eta)$. Then there exists $N_{1} \in \mathbb{N}$ and $\beta<1$ such that $S_{n}(p) \leq$ $n^{\beta}$ for all $n \geq N_{1}$, and there exists $N_{2} \in \mathbb{N}$ such that $\theta_{n}(p) \leq \frac{1}{2}$ for all $n \geq N_{2}$. Combining this with (7) and using the chain rule for Dini derivatives gives

$$
D^{+} \log \theta_{n}(p) \leq-\frac{1}{2 d} n^{1-\beta}\left(1-\theta_{n}(p)\right) \leq-\frac{1}{4 d} n^{1-\beta},
$$

for all $n>N:=N_{1} \vee N_{2}$. Let $p_{1}:=\left(\tilde{p}_{c}(\eta)+p\right) / 2$. Integrating the above inequality between $p_{1}$ and $p$ and using (9) gives

$$
\log \theta_{n}(p) \leq \log \theta_{n}(p)-\log \theta_{n}\left(p_{1}\right) \leq-\frac{1}{4 d}\left(p-p_{1}\right) n^{1-\beta} .
$$

It follows that

$$
\theta_{n}(p) \leq \exp \left(-\frac{1}{8 d}\left(p-\tilde{p}_{c}(\eta)\right) n^{1-\beta}\right)
$$

It remains to improve the above stretched exponential decay to proper exponential decay. From the stretched exponential decay it follows that $S(p):=$ $\lim _{n \rightarrow \infty} S_{n}(p)<\infty$. Combining this fact with (7), and using that $\theta_{n}(p) \leq \frac{1}{2}$ for $n>N$, since $p>\hat{p}_{c}(\eta)$, gives

$$
D^{+} \log \theta_{n}(p) \leq-\frac{1}{4 d S(p)} n .
$$

From here the proof is similar as for the stretched exponential decay, and we conclude

$$
\theta_{n}(p) \leq \exp \left(-\frac{1}{8 d S(p)}\left(p-\tilde{p}_{c}(\eta)\right) n\right) .
$$

It follows that $\tilde{p}_{c}(\eta)=\hat{p}_{c}(\eta)$, and that Theorem 1 holds with $c_{p}:=\frac{1}{8 d S(p)}\left(p-\tilde{p}_{c}(\eta)\right) \wedge \sup \left\{C>0: \theta_{n}(p) \leq \exp (-C n)\right.$ for all $\left.n \leq N\right\}>0$. 
Remark A mean-field lower bound can often be obtained from the analysis of a differential inequality such as the above one, which would be $\theta(p) \geq c\left(\tilde{p}_{c}(\eta)-p\right)$ in our case, for $p<\tilde{p}_{c}(\eta)$, and some constant $c>0$, independent of $p$. This does not directly follow from the above analysis, since we have assumed $p \in\left(\tilde{p}_{c}(\eta), \hat{p}_{c}(\eta)\right)$ in the first part of the analysis. If instead we take $p<\hat{p}_{c}(\eta)$ such that $\theta(p)<1 / 2$, then we can still bound $1-\theta_{n}(p) \geq 1 / 2$, for $n$ large enough, and obtain the meanfield lower bound at (10). However, this bound is of little interest for the orthant model, since if $p<p_{c}, \mathcal{C}^{*}(0)=\mathbb{Z}^{d}$ almost surely, so that $\theta(p)=1$. On the other hand, if $p \in\left(\tilde{p}_{c}, \tilde{p}_{c}(\eta)\right)$ for some $\eta>0$, Corollary 1 implies that $\theta(p)=1$ as well. This leaves the interval $\left(p_{c}, \tilde{p}_{c}\right)$ to be considered, but we conjecture this interval to be empty.

\section{Proof of the Shape Theorem}

To prove Corollary 1, we modify the proof of Holmes and Salisbury [14] in the places where they require $p$ to be large. Their proof is structured in seven lemmas, two of which require a large $p$. The first of these is Lemma 1 of [14]. This lemma asserts the existence of $\theta>1$, such that for every $\eta \in[0,1)$, there exists $p_{0}=p_{0}(\eta, d)<1$, such that for $p>p_{0}$, there exists $c_{1}>0$ such that $\mathbb{P}_{p}\left(0 \longrightarrow\left(-n \mathbf{1}+\mathcal{K}_{\eta}\right)^{c}\right) \leq$ $c_{1} \theta^{-n d}$, for all $n \in \mathbb{N}$. In the remainder of their proof, this lemma is only used for the case $\eta=0$. Therefore, we can replace this lemma by Theorem 1 , and require $p>\tilde{p}_{c}$, instead of $p>p_{0}$.

The second lemma in the proof of Holmes and Salisbury which require large $p$ is Lemma 5 of [14]. We will prove this lemma for $p>\tilde{p}_{c}$, instead of for large $p$, using Theorem 1. To state this lemma, we let $u \in \mathbb{Z}^{d} \backslash \mathbb{Z} \mathbf{1}$, and fix $v \in \mathbb{R}^{d}$ such that $u \cdot v>0$ and $v \cdot \mathbf{1}=0$. We define the slab

$$
\Lambda_{u, v}(m, n):=\left\{z \in \mathbb{Z}^{d}: m u \cdot v \leq z \cdot v<n u \cdot v\right\}
$$

We are interested in the following three events. Let $A_{n}^{\prime}(M)$ be the event there exists a path starting in 0 and ending in a point $k \mathbf{1}+n u$ with $k<n \gamma(u)$ that hits $\Lambda_{u, v}(-\infty,-M)$. Let $A_{n}^{\prime \prime}(M)$ be the event there exists a path starting in 0 and ending in a point $k \mathbf{1}+n u$ with $k<n \gamma(u)$ that hits $\Lambda_{u, v}(M+n, \infty)$. Lastly, let $\hat{A}_{n}$ be the event that there is a path starting in 0 and ending in some point $k \mathbf{1}$, with $k<0$, and reaches $\Lambda_{u, v}(n, \infty)$. We will prove the following lemma regarding these events:

Lemma 1 Let $p>\tilde{p}_{c}$. There exists $c>0$, such that $\mathbb{P}_{p}\left(A_{n}^{\prime}(\lfloor c n\rfloor)\right.$ i.o. $)=$ $\mathbb{P}_{p}\left(A_{n}^{\prime \prime}(\lfloor c n\rfloor)\right.$ i.o. $)=\mathbb{P}_{p}\left(\hat{A}_{n}(\lfloor c n\rfloor)\right.$ i.o. $)=0$.

We will prove the above lemma for the event $A_{n}^{\prime}(\lfloor c n\rfloor)$, the other two events can be proven similarly. The event $A_{n}^{\prime}(\lfloor c n\rfloor)$ is shown in Fig. 3. Let $p>\tilde{p}_{c}$. By the definition of this critical point there exists $\eta>0$ such that $p>\tilde{p}_{c}(\eta)$. We fix such an $\eta$. Let $c>0$, and let $M:=M(n):=\lfloor c n\rfloor$. We will choose the precise value of 


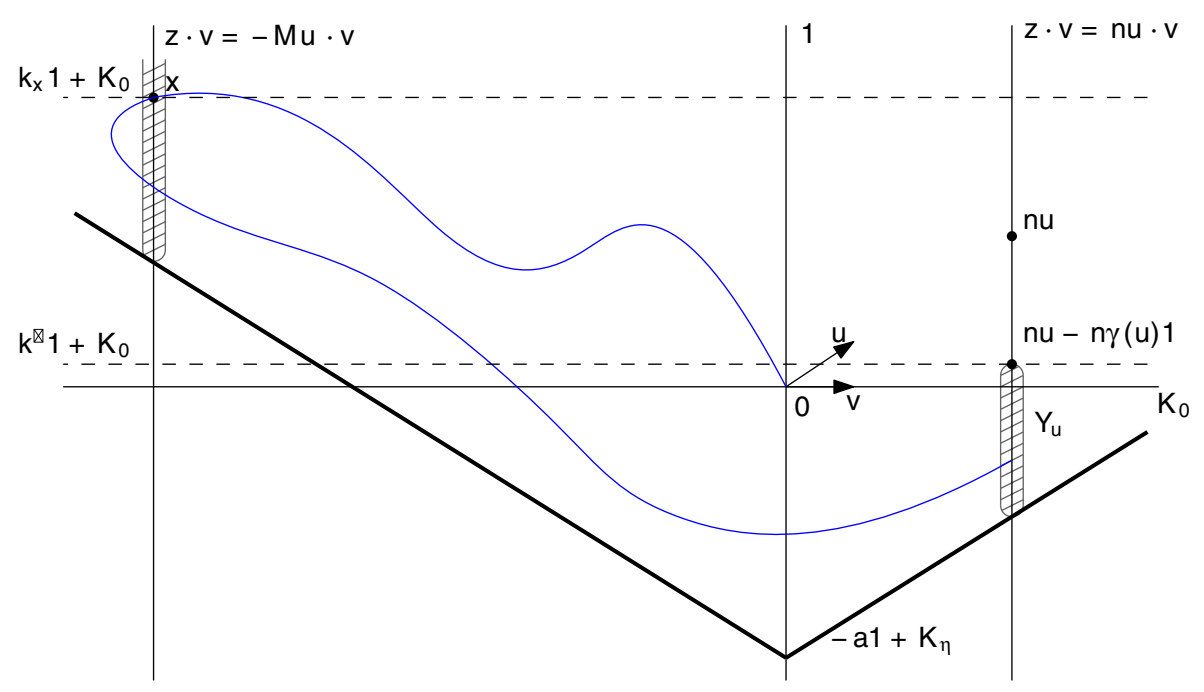

Fig. 3 When the event $A_{n}^{\prime}(M)$ occurs, there exists a path from 0 to $Y_{u}$ going through the shaded region

$c$ later on. Let $a>0$ and suppose $\mathcal{C}_{0}^{*} \subseteq-a \mathbf{1}+\mathcal{K}_{\eta}$. If $A_{n}^{\prime}(M)$ occurs, there exists $x \in \mathbb{Z}^{d}$ satisfying

$$
\left\{\begin{array} { r l } 
{ ( x + a \mathbf { 1 } ) \cdot \mathbf { 1 } } & { \geq \eta \| x + a \mathbf { 1 } \| _ { 1 } , } \\
{ x \cdot v } & { = - M u \cdot v , }
\end{array} \Longrightarrow \left\{\begin{array}{l}
x \cdot \mathbf{1} \geq-2 d a+\eta\|x\|_{1}, \\
x \cdot v=-M u \cdot v,
\end{array}\right.\right.
$$

such that $0 \longrightarrow x$, and $x \longrightarrow y$, with $y=k \mathbf{1}+n u$ for some $k<n \gamma(u)$. Since the $L^{1}$-norm is equivalent to the $L^{2}$-norm, and since the $L^{2}$-norm is invariant under an orthonormal basis change, it follows from the above equation that $\|x\|_{1} \geq c_{0} M$, for some constant $c_{0}=c_{0}(u, v)>0$. Combining this with the above inequality gives

$$
x \cdot \mathbf{1} \geq-2 d a+c_{0} \eta M .
$$

Define $k_{x}:=x \cdot \mathbf{1} / d$, so that $x \in k_{x} \mathbf{1}+\mathcal{K}_{0}$. Then the above inequality implies

$$
k_{x} \geq-2 a+\frac{c_{0} \eta M}{d} .
$$

We define the set

$$
Y_{u}:=\left\{y \in \mathbb{Z}^{d}: y=k \mathbf{1}+n u, \text { with } k<n \gamma(u)\right\} .
$$

We use Theorem 1 and the union bound to obtain

$$
\begin{aligned}
\mathbb{P}_{p}\left(A_{n}^{\prime}(M)\right) & \leq \exp \left(-c_{p} a\right)+\sum_{k_{x} \geq-2 a+c_{0} \eta M / d}^{\infty} \mathbb{P}_{p}\left(\exists x \in \Lambda_{u, v}(-\infty,-M): x \cdot \mathbf{1}=d k_{x}, 0 \longrightarrow x, x \longrightarrow Y_{u}\right) \\
& \leq \exp \left(-c_{p} a\right)+\sum_{k_{x} \geq-2 a+c_{0} \eta M / d}^{\infty} \sum_{\substack{x \in \Lambda_{u, v}(-\infty,-M), x \cdot \mathbf{1}=d k_{x}}}^{\infty} \mathbb{P}_{p}\left(x \longrightarrow Y_{u}\right) .
\end{aligned}
$$


We define $k^{*}:=k^{*}(n):=n(\gamma(u)+u \cdot \mathbf{1} / d)$. With this choice, it follows that $y \in\left(k^{*} \mathbf{1}+\mathcal{K}_{0}\right)^{c}$ for all $y \in Y_{u}$, and all $n \in \mathbb{N}$. We can now use translation invariance to bound

$\mathbb{P}_{p}\left(x \longrightarrow Y_{u}\right) \leq \mathbb{P}_{p}\left(x \longrightarrow\left(k^{*} \mathbf{1}+\mathcal{K}_{0}\right)^{c}\right)=\mathbb{P}_{p}\left(x-k_{x} \mathbf{1} \longrightarrow\left(-\left(k_{x}-k^{*}\right) \mathbf{1}+\mathcal{K}_{0}\right)^{c}\right)$.

We now fix

$$
c:=\left(\frac{d \gamma(u)+u \cdot \mathbf{1}}{c_{0} \eta}+1\right) \vee 1 .
$$

Let $k^{\prime} \geq 0$ such that $k_{x}=-2 a+\frac{c_{0} \eta M}{d}+k^{\prime}$. It holds, that

$$
\begin{aligned}
k_{x}-k^{*} & \geq-2 a+\frac{c_{0} \eta}{d}\left\lfloor n\left(\frac{d \gamma(u)+u \cdot \mathbf{1}}{c_{0} \eta}+1\right)\right\rfloor+k^{\prime}-n\left(\gamma(u)+\frac{u \cdot \mathbf{1}}{d}\right) \\
& \geq-2 a+\frac{c_{0} \eta}{d}(n-1)+k^{\prime}=: f\left(n, k^{\prime}\right) .
\end{aligned}
$$

It follows, that

$$
\mathbb{P}_{p}\left(A_{n}^{\prime}(M)\right) \leq \exp \left(-c_{p} a\right)+\sum_{k^{\prime}=0}^{\infty} \sum_{\substack{x \in \Lambda_{u, v}(-\infty,-M), x \cdot \mathbf{1}=-2 d a+c_{0} \eta M+d k^{\prime}}} \mathbb{P}_{p}\left(x-k_{x} \cdot \mathbf{1} \longrightarrow\left(-f\left(n, k^{\prime}\right) \mathbf{1}+\mathcal{K}_{0}\right)^{c}\right)
$$

Combining $x \cdot \mathbf{1}=-2 d a+c_{0} \eta M+d k^{\prime}$ with (11), shows that

$$
\|x\|_{1} \leq c_{0} M+\frac{d k^{\prime}}{\eta} .
$$

Using another union bound, translation invariance, and Theorem 1, we find

$$
\begin{aligned}
\mathbb{P}_{p}\left(A_{n}^{\prime}(M)\right) & \leq \exp \left(-c_{p} a\right)+\sum_{k^{\prime}=0}^{\infty}\left|\left\{x \in \mathbb{Z}^{d}:\|x\|_{1} \leq c_{0} M+\frac{d k^{\prime}}{\eta}\right\}\right| \mathbb{P}_{p}\left(0 \longrightarrow\left(-f\left(n, k^{\prime}\right) \mathbf{1}+\mathcal{K}_{0}\right)^{c}\right) \\
& \leq \exp \left(-c_{p} a\right)+\sum_{k^{\prime}=0}^{\infty}\left(2 c_{0} c n+2 \frac{d k^{\prime}}{\eta}\right)^{d} \exp \left(-c_{p} f\left(n, k^{\prime}\right)\right) .
\end{aligned}
$$

We now take

$$
a:=a(n):=\frac{c_{0} \eta}{4} n
$$

so that

$$
f\left(n, k^{\prime}\right)=-2 a+\frac{c_{0} \eta}{d}(n-1)+k^{\prime}=c_{0} \eta\left(\frac{n}{2}-1\right)+k^{\prime}
$$

A careful examination of (12) shows that the sum over $k^{\prime}$ converges for all $n \in$ $\mathbb{N}$, and that the result is summable with respect to $n$, so that by the Borel-Cantelli lemma $\mathbb{P}_{p}\left(A_{n}^{\prime}(\lfloor c n\rfloor)\right.$ i.o. $)=0$. The same result can be proven similarly for the events $A_{n}^{\prime \prime}(\lfloor c n\rfloor)$ and $\hat{A}_{n}(\lfloor c n\rfloor)$, and we omit the proof.

Acknowledgments I thank Matija Pasch for insightful discussions on the topic, as well as for useful comments on the manuscript.

Funding Open Access funding enabled and organized by Projekt DEAL. 
Open Access This article is licensed under a Creative Commons Attribution 4.0 International License, which permits use, sharing, adaptation, distribution and reproduction in any medium or format, as long as you give appropriate credit to the original author(s) and the source, provide a link to the Creative Commons licence, and indicate if changes were made. The images or other third party material in this article are included in the article's Creative Commons licence, unless indicated otherwise in a credit line to the material. If material is not included in the article's Creative Commons licence and your intended use is not permitted by statutory regulation or exceeds the permitted use, you will need to obtain permission directly from the copyright holder. To view a copy of this licence, visit http://creativecommons.org/licenses/by/4.0/.

\section{References}

1. Aizenman, M., Barsky, D.J.: Sharpness of the phase transition in percolation models. Comm. Math Phys. 108(3), 489-526 (1987)

2. Beekenkamp, T.: Sharp phase transitions in percolation models. In preparation (2021)

3. Beekenkamp, T.: Sharpness of the phase transition for the corrupted compass model on transitive graphs. Indagationes Mathematicae New Series 32(3), 736-744 (2021)

4. Dereudre, D., Houdebert, P.: Sharp phase transition for the continuum Widom-Rowlinson model Annales de l'Institut Henri Poincaré. Probabilités et Statistiques 57(1), 387-407 (2021)

5. Duminil-Copin, H., Goswami, S., Rodriguez, P.-F., Severo, F.: Equality of critical parameters for percolation of Gaussian free field level-sets. arXiv:2002.07735 (2020)

6. Duminil-Copin, H., Raoufi, A., Tassion, V.: Exponential decay of connection probabilities for subcritical Voronoi percolation in $\mathbb{R}^{d}$. Probab. Theory Relat. Fields, 1-12 (2017)

7. Duminil-Copin, H., Raoufi, A., Tassion, V.: Sharp phase transition for the random-cluster and Potts models via decision trees. Ann. Math. 189(1), 75-99 (2019)

8. Duminil-Copin, H., Raoufi, A., Tassion, V.: Subcritical phase of $d$-dimensional poisson-boolean percolation and its vacant set. Annales Henri Lebesgue 3, 677-700 (2020)

9. Grimmett, G. Percolation, volume 321 of Grundlehren der Mathematischen Wissenschaften [Fundamental Principles of Mathematical Sciences], second edition. Springer, Berlin (1999)

10. Holmes, M., Salisbury, T.S.: Degenerate random environments. Random Structures \& Algorithms 45(1), 111-137 (2014)

11. Holmes, M., Salisbury, T.S.: Random walks in degenerate random environments. Can. J. Math. 66(5), 1050-1077 (2014)

12. Holmes, M., Salisbury, T.S.: Conditions for ballisticity and invariance principle for random walk in non-elliptic random environment. Electron. J. Probab. 22, 1-18 (2017)

13. Holmes, M., Salisbury, T.S.: Phase transitions for degenerate random environments. ALEA 18, 707725 (2021)

14. Holmes, M., Salisbury, T.S.: A shape theorem for the orthant model. The Annals of Probability 49(3), 1237-1256 (2021)

15. Hutchcroft, T.: New critical exponent inequalities for percolation and the random cluster model. Probability and Mathematical Physics 1(1), 147-165 (2020)

16. Menshikov, M.V.: Coincidence of critical points in percolation problems. Dokl. Akad. Nauk SSSR 288(6), 1308-1311 (1986)

17. Muirhead, S., Vanneuville, H.: The sharp phase transition for level set percolation of smooth planar Gaussian fields. Annales de l'Institut Henri Poincaré. Probabilités et Statistiques 56(2), 1358-1390 (2020)

18. O’Donnell, R.: Analysis of Boolean Functions. Cambridge University Press, Cambridge (2014)

19. O’Donnell, R., Saks, M., Schramm, O., Servedio, R.A.: Every decision tree has an influential variable. In: 46th Annual IEEE Symposium on Foundations of Computer Science, 2005, pp. 31-39. IEEE (2005)

Publisher's Note Springer Nature remains neutral with regard to jurisdictional claims in published maps and institutional affiliations. 